\title{
Function-Oriented Inclusive Design
}

\author{
Markus Modzelewski \\ Universität Bremen, 28359 \\ Bremen, Enrique-Schmidt-Straße \\ 5, Germany \\ Email:modze@tzi.de
}

\author{
Michael Lawo \\ Universität Bremen, 28359 \\ Bremen, Am Fallturm 1, Germany \\ Email:mlawo@tzi.de
}

\begin{abstract}
Technological advancements regarding functional capabilities of products affect product development processes. We observe the following: More functionality can be included in smaller devices. New devices are invented as hybrids between existing devices. Products can be individually adapted for end customers. Although the current product development processes already include contextual information about target customer groups and scenario of use, this information is strongly linked to single devices. We present a hierarchical superstructure above devices regarding functional capabilities able to categorize devices by functionality but also recommend devices for a set of functionalities.
\end{abstract}

\section{INTRODUCTION}

$\mathrm{R}$ ECENT technological advancements emerged new opportunities in product design. Devices e.g. "phablets" include functional aspects related to mobile phone but also tablets in a single unit (Segan 2012). Automotive entertainment systems can be used to make a call or browse the internet (Zeller et al. 2001; BMW AG n.d.). With these new concepts, new usability issues appeared creating a burden especially for elderly customers regarding the need to learn how to use such devices (Clarkson et al. 2003; Langdon \& Thimbleby 2010).

On the other hand, design guidelines and pre-experience of designers regarding usability issues are included in recommendation-driven product design as presented by the VICON project (Lawo et al. 2011; Modzelewski et al. 2012; Kirisci et al. 2012). The output of the VICON software solution ConVic ${ }^{1}$ is extracted by a set of target user models, typical environments and typical tasks of a product (Modzelewski 2014). Design recommendations are presented defining qualitative and quantitative issues that should be included into product design increasing the awareness of designers about product customers. The ontology hierarchy used so far for storage and inference focuses on single devices (i.e. mobile phones). In this paper we extend the functional aspects including a selection of functionalities and inference of devices which can represent each service.

\section{II.EXISTING Modifications IN PRODUCt DEVELOPMENT}

According to VDI 2221 the product development process can be seen as a phase based sequence of states and outputs (VDI-Gesellschaft Entwicklung Konstruktion Vertrieb 1993). In the beginning the specification of requirements consists of a detailed analysis of user needs and functional aspects which are aimed for realisation by a product design. An analysis of a requirement list produces a functional structure for product decision. Product design consists of a draft phase in which first sketches (not function oriented) are constructed. In the subsequent $\mathrm{CAD}$ phase first virtual prototypes in virtual environments are produced which lead to prototypes. This enables an iterative process until the final product.

Customer involvement methods as seen by Strang and Linnhoff-Popien present different approaches how to include end customers into product design (Strang \& LinnhoffPopien 2004). The most frequently used Quality Function Deployment (QFD) method represents an analytical approach for first design phases with the involvement of end users (Akao 2004). A conversion of consumer demands into quality characteristics is utilized for an iterative deployment of a design quality function describing a "relation" between consumer and product. End customers are only involved during specification and concept phases but not for prototype testing, which leads to the categorization of QFD as a design for type. The types design with and design by have the pro of involvement of end customers in more phases with the con of being more cost-intensive (in addition to other pros and cons).

If customer involvement does not focus on device structures but functions as presented by this paper, new possibilities appear regarding design creativity but also the handling and integration of user needs aiming (a more) inclusive design (Coleman \& Lebbon 2005; Dong et al. 2004; Newell \& Gregor 2000).

\footnotetext{
${ }^{1}$ Available at SourceForge (http://sourceforge.net/projects/convic)
} 


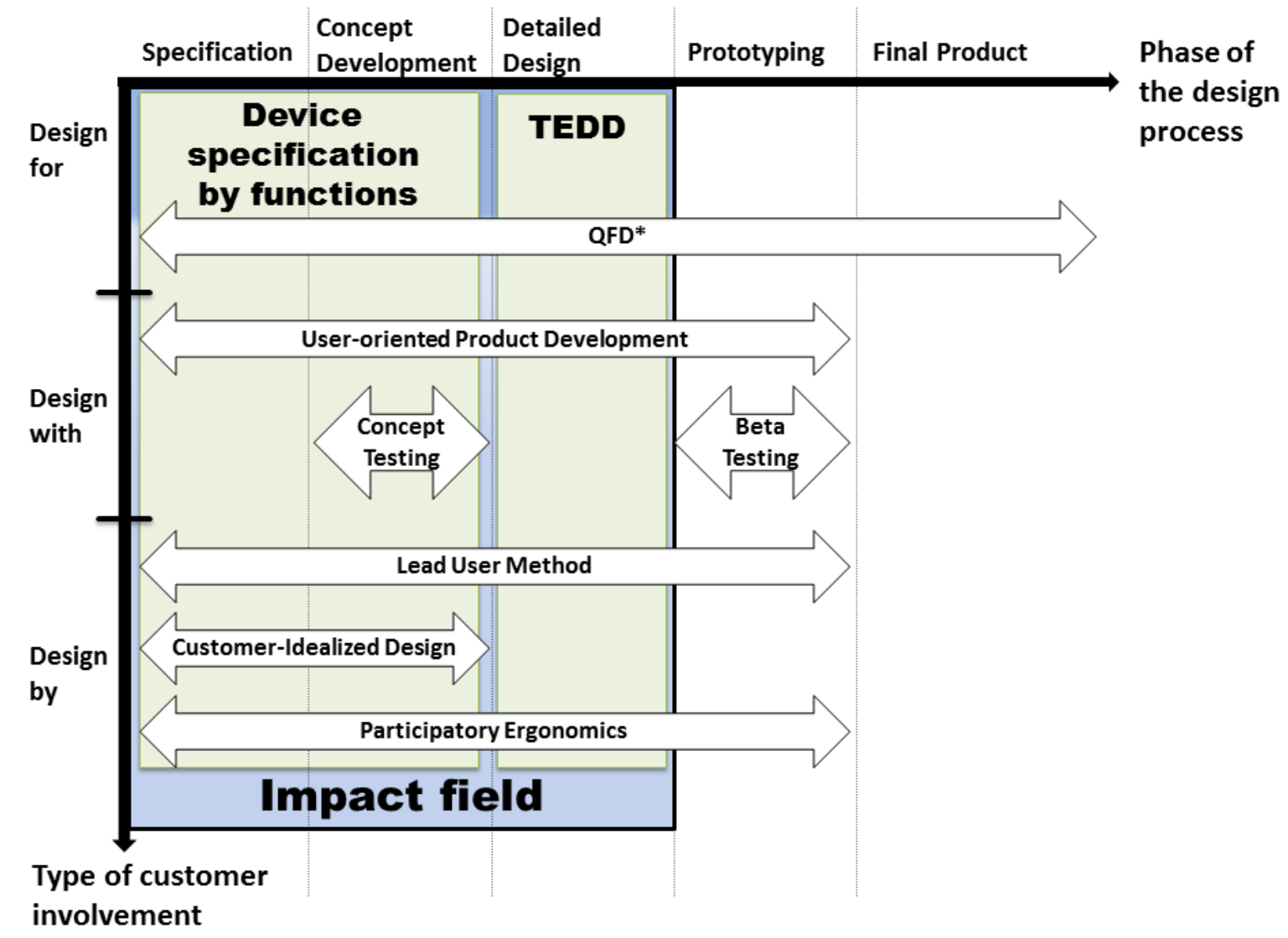

Figure 1: Impact field of function oriented approach on existing customer involvement methods.

- Quality function deployment

In QFD end users of a product are only involved before specification by forming relevant parameters and requirements of the product. If the design is not limited by a product but rather on functions, individual needs and capabilities of users can be integrated for more suitable products. As a result, inventions of new products based on end user requirements can be specified.

- User-oriented Product Development

This method focuses upon involvement of end customers after first concepts by direct involvement (design with users). Users are able to give feedback iteratively. If design is not design but function oriented, a use-analysis phase regarding functional possibilities is possible.

- Concept testing

After specification of products, this method aims an evaluation by customers regarding sketch drafts and first concepts. It should be supplemented with later evaluations (e.g. beta testing). If function oriented, concepts of solutions regarding functional parameters are tested.

- Beta testing

Beta testing focuses on product testing after first prototypes with end users. As products are already created in this phase, it should be supplemented by prior customer involvement strategies. From customer involvement perspective, this method starts when prototypes are already created, so the impact if a function oriented approach is applied is minimal.

\section{- Lead User Method}

"Lead Users" represent end customers facing needs that will be general in a future market. Design specifications until first prototypes are created by these users (supported by designers) to find appropriate solutions if functions cannot be executed accordingly. By orienting on functions instead of devices in this method, lead users are able to access a more sophisticated spectrum of solutions.

- Consumer idealized design

This method aims the generation of product designs by consumers with support of a facilitator as a group exercise. Participants of the exercise select representatives of target markets who create: A product design, a list of requirements of the product with focus to the target market and a record of reasons regarding design choices. If a design is not limited to a specific device, end user needs can be more directly integrated into a product.

- Participatory Ergonomics

The involvement of employees who participate in the production into the design process as designers but also as end users regarding product testing. This method especially aims the integration of experience from different groups knowing about limits regarding the production of a product. As production of a product mainly depends on hardware capabilities, a function-oriented approach would infer new production possibilities un-limiting device structures and enhancing (new) device inventions. 


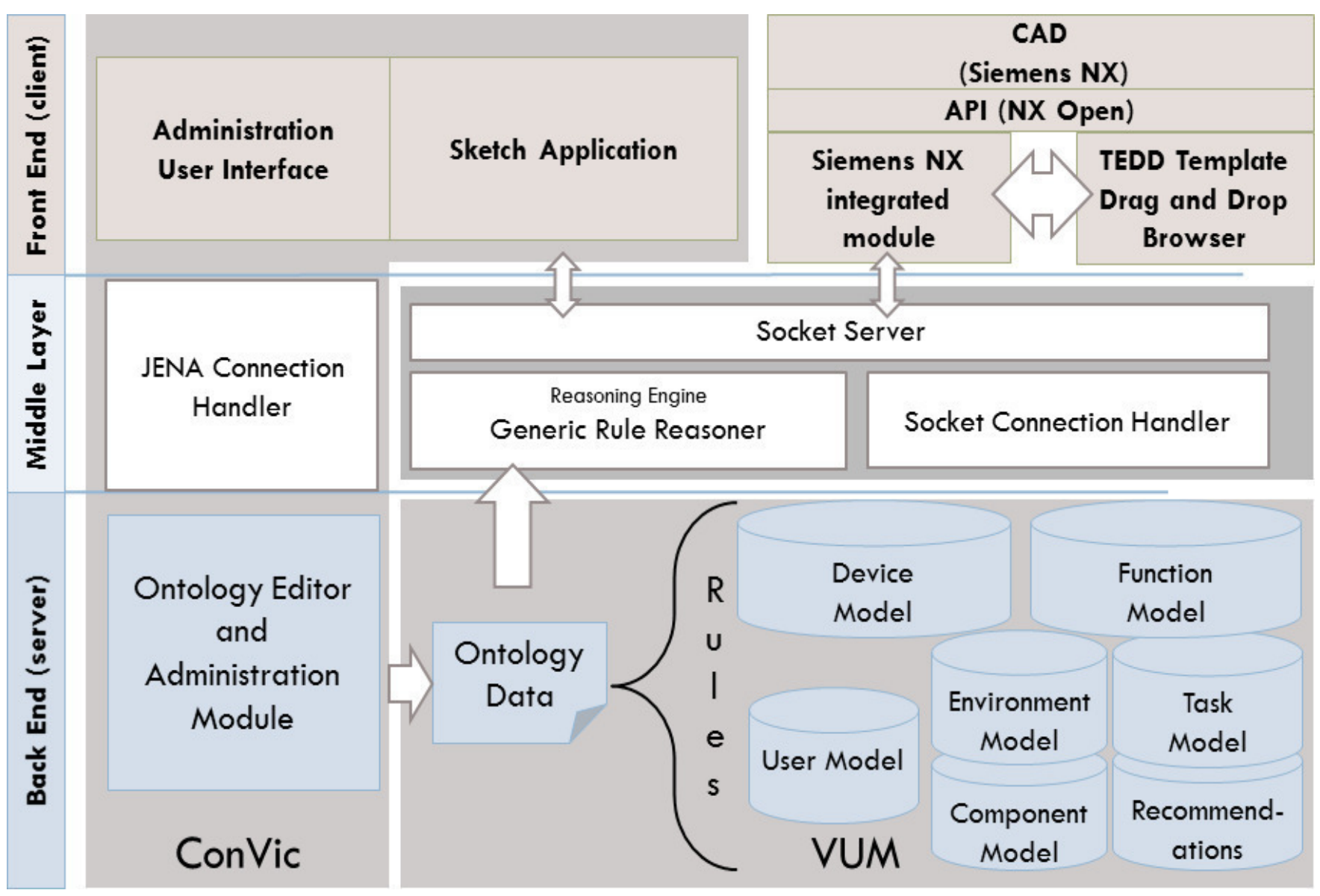

Figure 2: Device Model, Function Model and TEDD integrated into the VICON system architecture

The approach presented by this paper aims to integrate into two parts of product development (see Figure 1). In specification and concept development phase device specifications are described. By utilizing a model driven function superstructure designers (and end customers) are not limited by device structures but can orient on functional parameters. Other projects utilizing ontology approaches e.g. VERITAS are presented in (Modzelewski 2014; Poirson \& Delangle 2013).

\section{MODEL DRIVEN FUNCTION SUPERSTRUCTURE}

The ontology approach of ConVic utilizes Virtual User Models representing the scenario specification of the target product. It contains a User, Environment, Task, Component and Recommendation Model describing the target end customer group including impairments, typical environments with focus on e.g. background lighting, typical tasks for the target product, product components and qualitative and quantitative recommendations (Matiouk et al. 2013; Kirisci et al. 2011).

Device related models are Task and Recommendation Models. For each device, an ontology including both models is added to the initial ontology containing models of users, environments and components. To represent a variety of devices, the ontology containing Task and Recommendation Models is separated and can be replaced.

The pro of this approach is changing only the device related models for new products. Without this separation all task and recommendation instances must be recreated if producing a new device. Here we see redundancies. A less redundant approach would rely on functions instead of devices.

Figure 2 presents the approach integrated into the VICON system architecture. On ontology server side, device model and function model aim to implement the context of functional capabilities of devices into the existing approach. Existing models (User, Environment, Task, Component and Recommendation Model) can be re-used here as an initial ontology set. Already existing rules of can be reused but new rules must be added for the integration of function and device model merging all different models together (Modzelewski 2014). As only forward chain rules are implemented, the superstructure of function and device model on top of existing models can be implemented in addition to existing rules.

A function oriented approach does not focus on devices as the main target but on a set of related functions (see Table 1). It is possible for designers to choose functions, which the target device is able to perform, inferring a list of devices for realization. To create a new device, only a new instance as a member of the device model has to be created (see Table 2). 
An additional benefit of this separation is the representation of functional context information and the connection to the Component Model necessary for the dynamic presentation of CAD object templates (see section VI).

Table 1: Function Model predicates for ontology definition

\begin{tabular}{|ll|}
\hline Function Model & Description \\
\hline Predicate & Name of a single function \\
\hline Name & Unified ID for identification \\
\hline ID & $\begin{array}{l}\text { Brief description of the } \\
\text { function }\end{array}$ \\
\hline Description & $\begin{array}{l}\text { Sequence of Task Model } \\
\text { Instance IDs describing the } \\
\text { function }\end{array}$ \\
\hline TaskSequence & \\
\hline
\end{tabular}

The aim of the Function Model is to categorize functions individually with the purpose of building a relation between functional aspects of a design and task specific descriptions. The relation between functions and end user capabilities is made by the Task Model in which each instance categorizes problematic User Model Profiles. For example if a task can hardly be performed by users with manual dexterity impairments, a task instance consists of a value of "MD1, MD2" for the "Impairment" predicate (MD1 means mild, MD2 moderate manual dexterity impaired profile groups, see D2.2 of the VICON project).

Table 2: Device Model predicates for ontology definition

\begin{tabular}{|ll|}
\hline Device Model & Description \\
\hline Predicate & Name of a single device \\
\hline Name & Unified ID for identification \\
\hline ID & $\begin{array}{l}\text { Brief description of this } \\
\text { device }\end{array}$ \\
\hline Functions & $\begin{array}{l}\text { Sequence of Function } \\
\text { Model Instance IDs the } \\
\text { device is able to perform }\end{array}$ \\
\hline Components & $\begin{array}{l}\text { Sequence of Component } \\
\text { Model IDs, describing } \\
\text { recommended components } \\
\text { for CAD }\end{array}$ \\
\hline
\end{tabular}

The approach of the Device Model is similar. Devices can be described by the definition of each predicate for single instances. By creating this connection of function and device, designers are able to select a function and choose which device would be most suitable to represent this function. On the other side there is a need to create new devices, if a set of function is selected and no device is able to cover these.

\section{DEVICE SELECTION APPROACH}

The possibility to infer devices from functions represents a central unit in the modification of the current ontology approach. With addition of Function and Device Model new devices can be emerged if existing devices are not able to represent all selected functions.

Figure 3 presents an exemplary bonding between functions and devices regarding functionality. Users of the system are able to select a set of functions which can internally generate a set of tasks to integrate into the final ontology used. The selection defines instances of the general model are used for one specific target product. In the example there is no device available which is able to integrate a function set of "Make a call", "Read E-Mails" and "Watch TV". The closest device able to perform two of three functions is a mobile phone. Derived from this scenario, two issues can be stated: (1) It is necessary to create/invent a new device which is able to perform all three functions and (2) the new device should orient on mobile phones as they already are able to cover most of the functions.

Another question raised by this hypothetic scenario is why mobile phones are not able to realize the function to "Watch TV" inferring to include functions to devices if necessary.

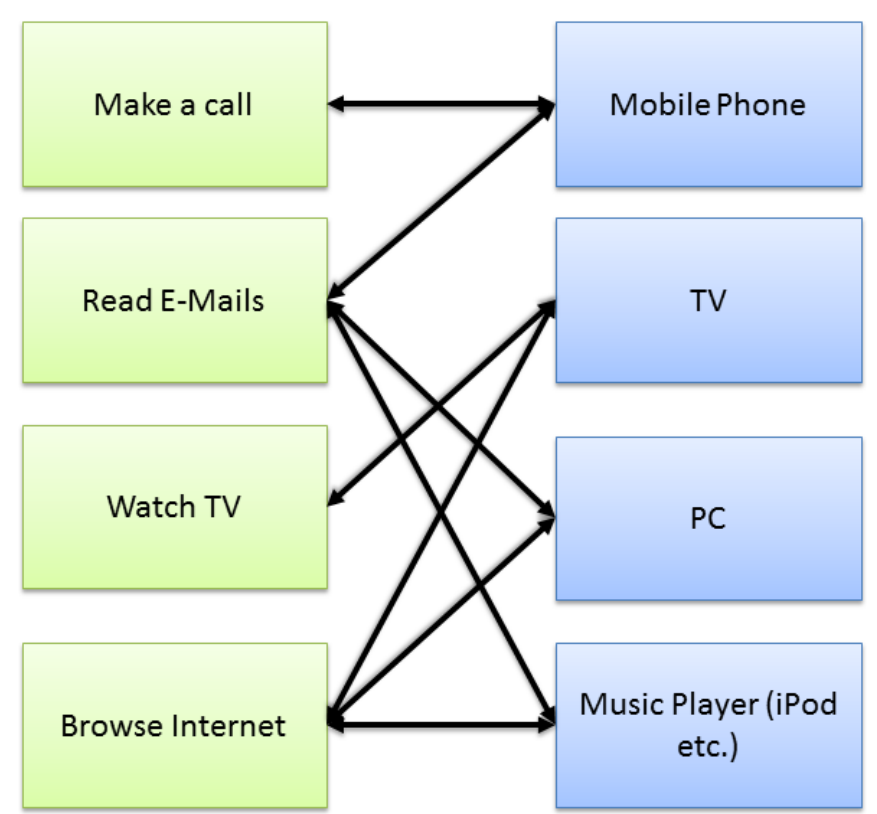

Figure 3: Exemplary bonds between functions and devices

The inference of the ontology created in the VICON project includes additional contextual information for bonding of each model regarding the output of recommendations based on a specific scenario (Lawo et al. 2011; Kirisci et al. 2012; Vicon Consortium 2012). If both Function and Device Model are included, predefined templates regarding a CAD model can be presented. An extension of the Component Model is necessary to include CAD objects including contextual data as brightness of a screen, noise of a speaker 
(templates). Designers will be able to choose from a set of template components to add or replace their current product design for products that can be used by an as wide group of population as possible.

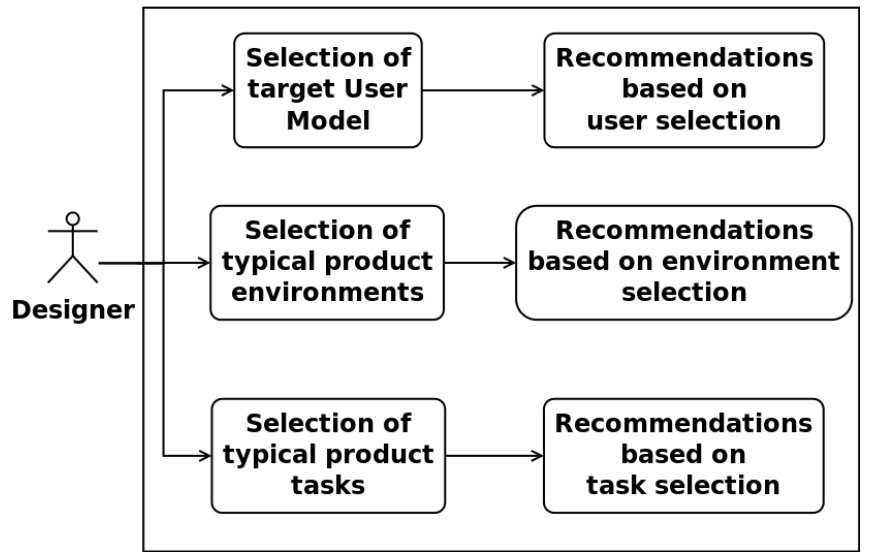

Figure 4: Abstract use case definition of designers using the support system ConVic

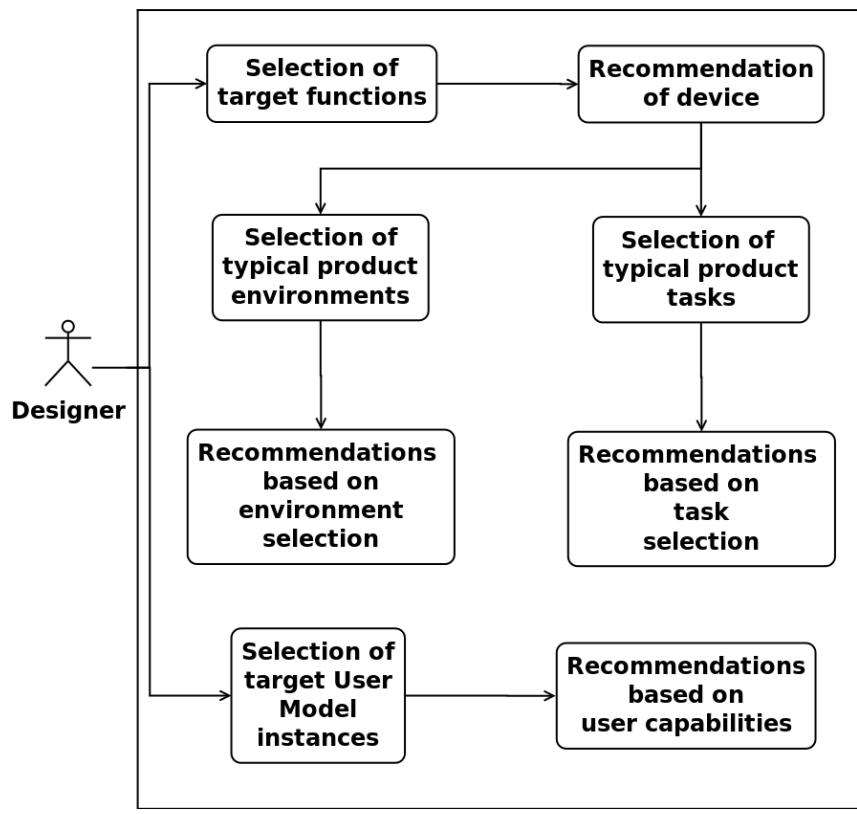

Figure 5: Abstract use case definition of designers related to functional aspects

The basic idea is to support designers through the complete product development process. Figure 4 presents a very abstract view of general input and output during the sketch design phase defining the selection of target users, typical product environment and typical tasks that can be performed by the target product. For the second phase the input of product components is added inferring the output of quantitative recommendations (Kirisci et al. 2011). The extension of a Function and Device Model enables this procedure to infer recommendations based on functional aspects for a more general view. Instead of the selection of environment and task specifications, designers are able to select functions.
Derived from these functions, scenario-related context can be applied including (one or more) environments and tasks.

In addition to the recommendation output as seen in Figure 5, it is also possible for designers to get CAD related templates of product components. An extension to the Component Model of CAD templates enables the possibility to generate from function selection a set of inclusively designed components.

\section{V.EXTENSION OF COMPONENT MODEL}

The component model used in ConVic does not include $\mathrm{CAD}$ related context information but component features (e.g. how many states does a component have) necessary for the presentation of qualitative and quantitative recommendations (Matiouk et al. 2013; Modzelewski 2014). An extension is needed regarding the inference of templates as defined in the previous chapter. The additional predicate contains the file path of a CAD file is utilized for the next step of template presentation. Instances of the component model are also inferred from recommendations directly, CAD templates can be produced by two designer selections. The current selection of target User Model identifying end user capabilities but also the selection of functions the device should be able to perform.

These templates include parametrical data as attributes to identify additional aspects regarding functionality of a component. Initial CAD objects do not include any contextual data. For example if a press button is created, inside of the $\mathrm{CAD}$ environment it is represented as a cube shape object. The representation of contextual data regarding functionality but also component related aspects set a basis for the improvements of product development processes.

Another additional pro is the simplification measure to create individual products for customers, if preferences of end customers regarding component functionalities can already be included during $\mathrm{CAD}$ phase.

\section{INCLUSIVE DESIGN TEMPLATES FOR CAD}

The utilization of the selection of functionalities is able to produce a set of inclusively designed templates for the CAD environment. Based on the target functionality selection ( Figure 5) the system is able to produce a set of components predefined in the Component Model for implementation in the current $\mathrm{CAD}$ environment Siemens $\mathrm{NX}^{2}$. The templates already include contextual information necessary for the adaptation during the CAD phase as seen in (Modzelewski 2014).

The conceptual tool TEDD (TEmplate Drag and Drop browser for inclusive design) presents a set of inclusively designed objects.

\footnotetext{
${ }^{2}$ http://www.plm.automation.siemens.com/de_de/products/nx/
} 


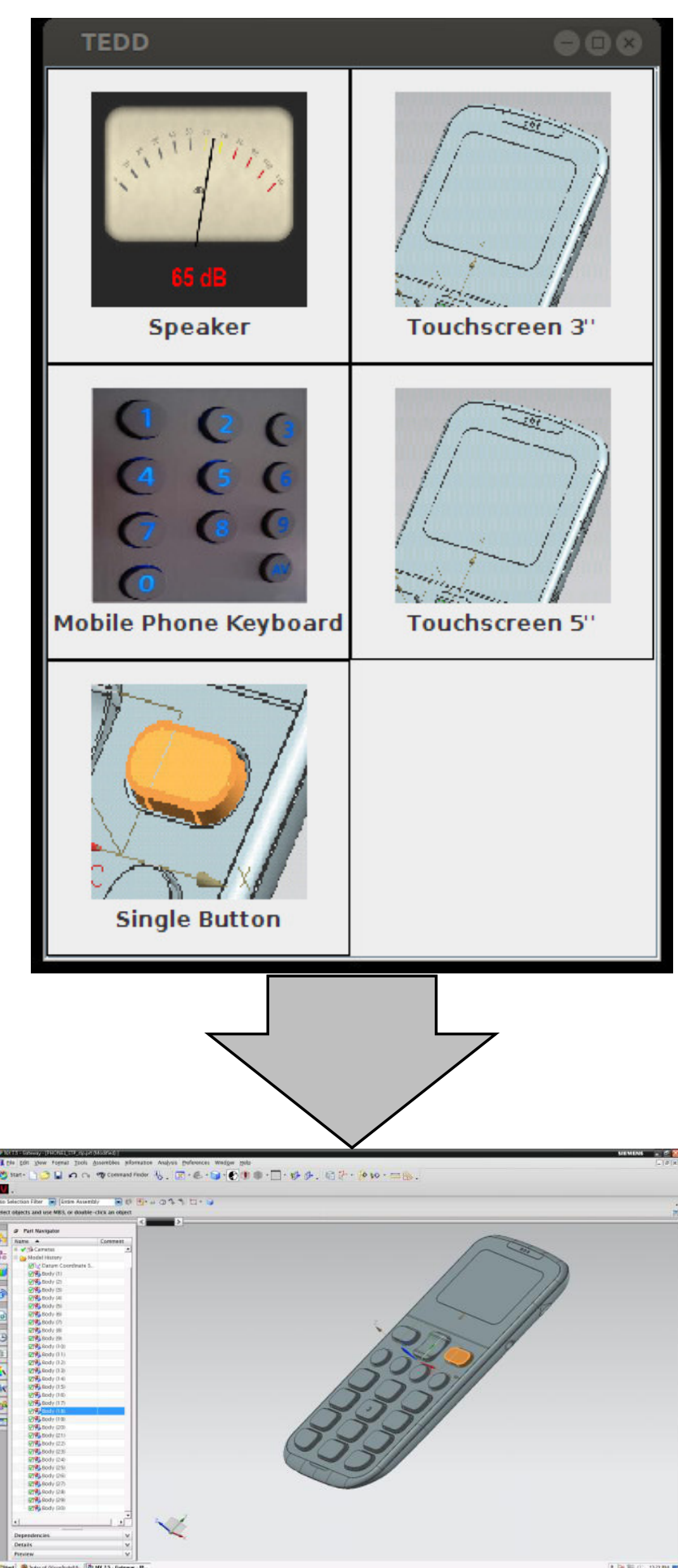

Figure 6: TEDD - Template browser for Drag \& Drop of inclusively designed components in CAD environment

Figure 6 presents an exemplary setup based on components necessary for the functionality to make a call. Using the selection of the end user (designer) of functions, a set of possible devices for each function is generated using the Functions predicate as seen in Table 2. In addition to existing qualitative and quantitative recommendations of the VI-
CON system, Users are able to include predefined templates and adjust them.

The following rule describes a simple rule based solution for connecting functions with devices by predicates as seen in (The Apache Software Foundation 2013). First two variables "? $x$ " and "? $y$ " are created including variables for their ID predicate. The predicate Functions is stored in the variable "?fun". By concatenating the variable "?funid" between “(.*)”, a regular expression is utilized which is always true, if the function ID is found within the "?fun" variable. The second concatenation is used for definition of the recommendation class containing all recommended devices for a single function.

(?x rdf:type Vicon:Function), (?x Vicon:FunctionID ?funid), (?y rdf:type Vicon:Device), (?y Vicon:DeviceID ?devid), , (?y Vicon:DeviceFunctions ?fun),

strConcat(“(.*)”, ?funid,"(.*”), ?reg), regex(?fun,?reg), strConcat("Vicon:FunctionReco_", ?funid, ?recoClass) -> (?y rdf:type ?recoClass).

By adding redundant components as several versions of touchscreens, the impact on creative process of design is as small as possible.

In the next step integration into Siemens NX CAD environment is aimed, utilizing the integrated knowledge-reuse module ${ }^{3}$.

\section{CONCLUSION}

This paper presented an approach and a tool for the generation of a set of inclusive design CAD templates based on the target functions of a product design. Furthermore a function and device model was introduced representing functional and device related aspects utilized for the generation of an optimal device configuration. The developed software is open source and accessible via the VICON project homepage (Vicon Consortium 2013). The usefulness of the approach was evaluated with eleven physical product designers. The results of this investigation with these designers as well as the outcome of an evaluation with forty-eight beneficiaries of products designed using the presented approach are published in (Modzelewski 2014).

\section{VIII.FUTURE WORK}

Regarding a more detailed functional design Stone and Wood (Stone \& Wood 2000) presented a functional basis regarding material, energy and signal. It would be interesting how and if it is necessary to represent each single aspect of functional design but also if this would be suitable to even ignore devices as an instance focussing only on functional aspects. An extension and population of the Component

\footnotetext{
${ }^{3}$ Siemens NX knowledge-reuse module:

https://www.plm.automation.siemens.com/de_de/products/nx/fordesign/knowledge-re-use/library.shtml\#lightview-close
} 
Model regarding the integration of CAD templates into the model is advantageous for the generation of recommended components. For this implementation a variety of CAD templates including contextual data is needed.

\section{REFERENCES}

Akao, Y., 2004. Quality function deployment: integrating customer requirements into product design, Productivity Press.

BMW AG, BMW Website - BMW Techniklexikon : Controller.

Clarkson, J. et al., 2003. A designer-centred approach. Inclusive design: Design for the whole population.

Coleman, R. \& Lebbon, C., 2005. Inclusive design. Helen Hamlyn Research Centre, Royal College of Art.

Dong, H., Keates, S. \& Clarkson, P., 2004. Inclusive design in industry: barriers, drivers and the business case. User-Centered Interaction Paradigms for Universal Access in the Information Society, pp.305-319.

Kirisci, P. et al., 2012. Supporting Inclusive Design of Mobile Devices with a Context Model. InTech Open Science.

Kirisci, P. et al., 2011. Supporting inclusive design of user interfaces with a virtual user model. Universal Access in Human-Computer Interaction. Users Diversity, pp.69-78.

Langdon, P. \& Thimbleby, H., 2010. Inclusion and interaction: Designing interaction for inclusive populations. Interacting with Computers, 22(6), pp.439-448.

Lawo, M. et al., 2011. Virtual User Models - Approach and first results of the VICON project. In P. Cunningham \& M. C. (Eds), eds. eChallenges e-2011 Conference Proceedings. IIMC International Information Management Corporation Ltd.

Matiouk, S. et al., 2013. Prototype of a Virtual User Modeling Software Framework for Inclusive Design of Consumer Products and User Interfaces. In Universal Access in Human-Computer Interaction. Design Methods, Tools, and Interaction Techniques for eInclusion. Springer, pp. 59-66.

Modzelewski, M., 2014. An ontology-based approach to achieve inclusive design support in the early phases of the product development process. Bremen: University of Bremen, Dissertation. Available at: http://nbnresolving.de/urn:nbn:de:gbv:46-00103662-13 [Accessed March 11, 2014].

Modzelewski, M. et al., 2012. Creative Design for Inclusion Using Virtual User Models. Computers Helping People with Special Needs, pp.288-294.

Newell, A.F. \& Gregor, P., 2000. User sensitive inclusive design - in search of a new paradigm. In Proceedings on the 2000 conference on Universal Usability. CUU '00. New York, NY, USA: ACM, pp. 39-44. Available at: http://doi.acm.org/10.1145/355460.355470.

Poirson, E. \& Delangle, M., 2013. Comparative analysis of human modeling tools.

Segan, S., 2012. Enter the Phablet: A History of PhoneTablet Hybrids.

Stone, R.B. \& Wood, K.L., 2000. Development of a Functional Basis for Design. Journal of Mechanical Design, 122(4), p.359. Available at: http://mechanicaldesign.asmedigitalcollection.asme.or g/article.aspx?articleid=1446060 [Accessed March 18, 2014].

Strang, T. \& Linnhoff-Popien, C., 2004. A context modeling survey. In Workshop Proceedings.

The Apache Software Foundation, 2013. Reasoners and rule engines: Jena inference support.

VDI-Gesellschaft Entwicklung Konstruktion Vertrieb, 1993. VDI 2221, Methodik zum Entwickeln und Konstruieren Technischer Systeme und Produkte. Verein Deutscher Ingenieure (Hrsg.): VDI-Handbuch Methodisches Konstruieren, Berlin.

Vicon Consortium, 2013. ConVic I Free Graphics software downloads at SourceForge.net. Available at: http://sourceforge.net/projects/convic/ [Accessed March 21, 2014].

Vicon Consortium, 2012. Project Deliverable 2.2 - Virtual User Model (Final release). Available at: http://www.vicon-project.eu.

Zeller, A., Wagner, A. \& Spreng, M., 2001. iDrive-Zentrale Bedienung im neuen 7er von BMW/iDrive-centralised operation in the new 7series of BMW. VDI-Berichte, (1646). 